\title{
Analysis on Wavelet Threshold Extracting Weak Signal in Deep Space Communication
}

\author{
Bo Zeng, Dezhi Li, Qing Guo ${ }^{+}$and Zhenyong Wang \\ Communication Research Center, Harbin Institute of Technology, Harbin, China, 150001
}

\begin{abstract}
The extremely long distance of deep space communication brings about the tough challenge that the received signal is weak. In order to overcome the problem of low signal to noise ratio (SNR) at the lowest price, we extract weak signal by means of wavelet thresholding method, in which the weak signal energy can be gathered in a few of sparse wavelet transform coefficients, and the energy of noise will spread to all the coefficients, which means that the output carrier-to-noise-ratio (CNR) can be effectively improved by wavelet thresholding. Additionally, the influence of the wavelet decomposition level on the output CNR and the correlation coefficient of the real signal and estimated signal is extensively discussed by computer simulations. Based on the discussions, we give the suggestions on practical applications of wavelet thresholding method.
\end{abstract}

Keywords: performance, wavelet threshold, extracting weak signal, deep space communication.

\section{Introduction}

The deep space exploration plays an increasingly important role in exploring space resources since entering the 21st century. Meanwhile it brings numerous issues on the deep space communication [1]. Long distance transmission of the deep space communication makes the energy of the received signal be small to meet the requirement of high speed information transmission. Communication distance of the deep space communication is one hundred million kilometers magnitude in general [2]. The communication link is so long that will cause great signal attenuation, which thus can make the received signal strength is very weak and the signal energy is often lower than the energy of the noise. The transmission rate of the information will be very low if the received signal is directly decoded. However, with the development of deep space communication, the rate of information transmission has been higher and higher [3]. In this trend, the SNR of received signal is lower than that of receiver decoding threshold. For overcoming the problem of low SNR, many methods have been proposed to improve the energy per bit of the received signal, such as increasing the transmit power or enlarging the antenna size, however, which is proved to bring great costs. The bigger the antenna size is, the greater the gain of the communication link is [4]. But it will increase the weight of the antenna, which will also increase the difficulty of the launch of the spacecraft [5], [6].

The above methods can not satisfy the requirement of the current deep space communications. An efficient method to extract the weak signal is to use the weak signal extraction algorithm, which can be implemented in a short term. There are many ways to extract the weak signals, such as stochastic resonance method, chaos theory and so on. The stochastic resonance method is very effective for the signal processing of the signal with the noise of tens of $\mathrm{Hz}$. But it can only handle the signals at most $1 \mathrm{MHz}$, however, which is not suitable for the extraction of weak signals of deep space communication where the IF signal reaches tens of MHz. Despite chaos theory can extract the weak signal [7] from the chaotic background by reconstructing the phase space of chaotic background noise, the method is not applicable because the signal of deep space communication doesn't belong to chaotic signal.

\footnotetext{
+ Corresponding author. Tel.: +86 13604806972.

E-mail address: qguo@hit.edu.cn.
} 
Considering a variety of weak signal extraction algorithm, in which the weak signal can be gathered in a few of sparse wavelet transform coefficients, and the energy of the noise will spread to all of the coefficients [8]. So we can set a threshold, and the coefficients which are lower than the threshold will be set to zero. After processing the signal, it will be able to subtract noise from the weak signal, which is very conductive to the extraction of weak signal of the deep space communication. Thus, the performance of the wavelet threshold method to extract the signal of deep space communication is discussed in this paper.

\section{Theory on Wavelet Thresholding Method}

Wavelet transform, originally proposed by the French physicist Morlet and Grossmann, was intended to facilitate the analysis of geological data [9]. The essence of wavelet transform is to decompose the timedomain signal into the linear combination of wavelet function. The expression of wavelet transform for timedomain signal $f(t)$ is $W(J, k)=\int_{R} f(t) \psi_{-J, k} d t$, where $\psi_{-J, k}=2^{-J / 2} \psi\left(2^{-J} t-k\right)$ is the wavelet function. In this paper, the weak signal extraction and analysis is based on the following model.

$$
y=\chi(t)+n
$$

where $\chi(t)$ is the ideal received signal, $n$ is the additive white Gaussian noise (AWGN) and $y$ is the received signal which contains noise.

The wavelet transform can extract the weak signal mainly because the wavelet function has the highorder vanishing moment, because of which the wavelet coefficients corresponding to the signal become sparse and energy is more concentrated. If the wavelet function $\psi(x)$ meets the following formula,

$$
\int_{R} t^{k} \psi(t) \mathrm{d} t=0,0 \leq k<m
$$

thus the wavelet function $\psi(x)$ has the vanishing moment of $m$ orders.

Assuming the wavelet function $\psi(x)$ has the vanishing moment of $L$ orders, the time-domain signal $\chi(t)$ is carried out at the place $t=2^{-j} k$ by Taylor series expansion.

$$
\chi(t)=\chi\left(2^{-J} k\right)+\chi^{\prime}\left(2^{-J} k\right)\left(t-2^{-J} k\right)+\cdots+\frac{1}{(L-1) !} \chi^{(L-1)}\left(2^{-J} k\right)\left(t-2^{-J} k\right)^{L-1}+\left(t-2^{-J} k\right)^{L} R(t)
$$

The value of the wavelet decomposition coefficient of the signal $\chi(t)$ can be expressed as below.

$$
\left|\left\langle\chi, \psi_{-J, k}\right\rangle\right|=\left|\int\left(t-2^{-J} k\right)^{L} R(t) 2^{J / 2} \psi\left(2^{J} t-k\right) d t\right| \leq C 2^{-J(L-1 / 2)} \int|y|^{L}|\psi(y)| d y
$$

The BPSK phase modulation is used in the information data in deep space communication. The signal is relatively smoother than the noise except the phase jump point and the boundary point. Weak signal energy can be gathered into sparse coefficient by the wavelet transform [10]. The wavelet coefficients of white noise are distributed in each coefficient, and the energy of the noise is dispersive relatively. So it is helpful for distinguishing weak signals and noises, and also conducive to the extraction of weak signal. And this is well suited for the situation of energy of weak signal lower than noise energy in deep space communication [11]. Since the wavelet transform concentrates the signal energy on the sparse coefficients, the majority of coefficients of the larger amplitude is retained while that of smaller amplitude is abandoned. Namely, we can set a threshold, and the coefficients which are lower than the threshold will be set to zero. So the weak signal can be extracted from this idea.

\section{Analysis with Wavelet Extracting Weak Signal}

The ability of extracting the weak signal is directly characterized by the CNR of the output signal of the deep space weak signal through the weak signal extraction system. In this paper, the CNR of the output signal is one of the reference indexes. Its definition can be described as the equation (5).

$$
C N R=10 \cdot \lg \left(\sum_{i=1}^{N}\left|\chi\left(t_{i}\right)\right|^{2} / \sum_{i=1}^{N}\left|\chi_{e}\left(t_{i}\right)-\chi\left(t_{i}\right)\right|^{2}\right)
$$


Taking into account the IF signal is extracted, carrier tracking is needed for extracting the carrier signal at the same time. Then the IF signal also need to be demodulated. Therefore, in order to ensure the normal communication, the signal distortion can't be too large. Otherwise we can't do the subsequent operation. In this paper, the deformation measurement of output signal is characterized by the correlation coefficient between output signal and real signal [12]. And correlation coefficient between output signal and real signal can be expressed as the equation (6).

$$
\operatorname{Corr}=\sum_{i=1}^{N}\left(\chi\left(t_{i}\right)-\bar{\chi}\right)\left(\chi_{e}\left(t_{i}\right)-\bar{\chi}_{e}\right) / \sqrt{\sum_{i=1}^{N}\left(\chi\left(t_{i}\right)-\bar{\chi}\right)^{2} \cdot \sum_{i=1}^{N}\left(\chi_{e}\left(t_{i}\right)-\bar{\chi}_{e}\right)^{2}}
$$

where $N$ is Signal sampling points, and $\chi\left(t_{i}\right)$ is the value of the $i$-th ideal received signal sample point, and $\chi_{e}\left(t_{i}\right)$ is the estimation of the $i$-th ideal received signal sample. Both $\bar{\chi}$ and $\bar{\chi}_{e}$ are the average of $N$ point data. And their expressions are as follows.

$$
\begin{gathered}
\bar{\chi}=\sum_{i=1}^{N} \chi\left(t_{i}\right) / N \\
\bar{\chi}_{e}=\sum_{i=1}^{N} \chi_{e}\left(t_{i}\right) / N
\end{gathered}
$$

This article selects soft threshold function method to extract weak signals [13]. The influence of noise in signal can be removed by soft threshold method processing wavelet coefficients of weak signal. The expression of soft threshold function can be described as the following equation [14].

$$
\hat{\omega}=\left\{\begin{array}{c}
0,|v|<\lambda \\
\operatorname{sgn}(v) \cdot(|v|-\lambda),|v| \geq \lambda
\end{array}\right.
$$

where $v$ is the high frequency decomposition coefficient of noisy signal in wavelet domain and $\lambda$ is the set threshold. And $\hat{\omega}$ is the wavelet coefficient of noisy signal after the threshold processing.

The selection of threshold determines the performance of weak signal extraction. In the following the selection method of optimal threshold in wavelet threshold method is presented. In order to make the mean square error (MSE) between the real signal and the output signal as small as possible, we need to select the appropriate threshold. Because both the signal and the noise are random signals, the selection of the optimal threshold will be the minimum of the error of the output signal and the real signal in the statistical sense. Namely, the formula (10) obtains the minimum value [15].

$$
M S E=\left\|\chi-\chi_{e}\right\|^{2}
$$

where $\chi$ is the real signal, and $\chi_{e}$ is the estimation signal. According to the Parsevel's theorem, we can obtain

$$
\left\|\chi_{e}-\chi\right\|^{2}=\|\hat{\omega}-\omega\|^{2}
$$

Combined with the formula (10), MSE can be expressed as the equation (12).

$$
M S E=\|\hat{\omega}-\omega\|^{2}
$$

Difference between real wavelet coefficients and decomposition coefficients of noisy signals is denoted as $y(v)$.

$$
y(v)=\hat{\omega}-v
$$

For the wavelet coefficients of the $i$-th sample point, the statistical average of the variance between real signal and estimated signal can be described as below. 


$$
\begin{aligned}
E\left\{\omega_{i}-\hat{\omega}_{i}\right\}^{2} & =E\left\{y\left(v_{i}\right)+v_{i}-\omega_{i}\right\}^{2} \\
& =E\left\{\left(v_{i}-\omega_{i}\right)^{2}+2\left(v_{i}-\omega_{i}\right) y\left(v_{i}\right)+y^{2}\left(v_{i}\right)\right\} \\
& =1+2 E\left\{\nabla_{i} y\left(v_{i}\right)\right\}+E\left\{y^{2}\left(v_{i}\right)\right\}
\end{aligned}
$$

where $\nabla_{i}\left(y\left(v_{i}\right)\right)=\frac{\partial y\left(v_{i}\right)}{\partial v_{i}}$. If the threshold function is continuous, the MSE of all $N$ points is count to calculate the statistical average of it after the weak signal is extracted by the threshold method.

$$
E\|\hat{\omega}-\omega\|^{2}=N+E\left\{\|y(v)\|^{2}+2 \nabla y(v)\right\}
$$

where $\|y(v)\|^{2}=y(v) \cdot y(v)=\sum_{i=1}^{N} y^{2}\left(v_{i}\right)$ and $\nabla y(v)=\sum_{i=1}^{N} \nabla_{i}\left(y\left(v_{i}\right)\right)=\sum_{i=1}^{N} \frac{\partial y\left(v_{i}\right)}{\partial v_{i}}$. Combined with the formula (9), we can obtain that

$$
\begin{gathered}
\|y(v)\|^{2}=\sum_{i=1}^{N} y^{2}\left(v_{i}\right)=\sum_{i=1}^{N}\left(\left|v_{i}\right| \wedge \lambda\right)^{2} \\
\nabla y(v)=\sum_{i=1}^{N} \frac{\partial y\left(v_{i}\right)}{\partial v_{i}}=\Theta\left\{i:\left|v_{i}\right| \leq \lambda\right\} \\
E\|\hat{\omega}-v\|^{2}=N-2 \cdot \Theta\left\{i:\left|v_{i}\right| \leq \lambda\right\}+\sum_{i=1}^{N}\left(\left|v_{i}\right| \wedge \lambda\right)^{2}
\end{gathered}
$$

where $N$ is the number of the coefficients of the signal in the wavelet domain. $\left|v_{i}\right| \wedge \lambda$ is the minimum value between the $\left|v_{i}\right|$ and $\lambda$. And $\Theta\{\cdot\}$ represents a total number of elements which meet the condition after the colon.

From the equation (18), we can obtain that $\Theta\left\{i:\left|v_{i}\right| \leq \lambda\right\}$ remains unchanged and $\sum_{i=1}^{N}\left(\left|v_{i}\right| \wedge \lambda\right)^{2}$ decreases with the decrease of the $\lambda$ accordingly when the threshold $\lambda$ changes between the two absolute values of the adjacent coefficients. Hence the statistical average of MSE obtains the minimal on the absolute value of the wavelet coefficient.

The vanishing moment of the wavelet function determines the degree of the signal energy accumulation in the wavelet domain after the weak signal is decomposed. The higher the vanishing moment is, the more concentrated the signal energy and the better the signal extraction effect is. However, higher order vanishing moment brings more computation, which causes big problems that it need more time to calculate and that it is not suitable for deep space communication because of its special environment.

\section{Numeral Results and Discussions}

In this section, the ability of extracting deep space weak signal by wavelet threshold is simulated and analyzed. And some results are also meaningful for practical engineering. This paper chooses Daubechies wavelet to extract weak signal in deep space communication. In section 3, we have discussed that the performance of extracting signal is influenced by the vanishing moment of the wavelet function. So we need to select the wavelet function with appropriate vanishing moment. The relationship between the wavelet vanishing moment and the CNR of the output signal is given in the Fig. 1.

From the Fig. 1, it can be seen that in the case that the CNR of the input signal is determined, the CNR of output signal increases with the increase of the vanishing moment of the wavelet function. The rise of the output CNR will slow down gradually, and the increase rate of the output signal CNR tends to be 0 when the vanishing moment of wavelet function is more than 10. The relationship between the wavelet vanishing moment and the correlation coefficient of the real signal and the output signal is given by the Fig. 2. From the Fig. 2, in the case that the CNR of the input signal is determined, the output signal and real signal are 
more relevant with the increase of the vanishing moment of the wavelet function. And the correlation coefficient tends to be stable when the vanishing moment of wavelet function is more than 10 .

From the Fig. 3 and the Fig. 4, it can be seen that the output CNR and the correlation coefficient are both gradually increasing with the rise of the number of the decomposition layers at the condition that the CNR of the input signal is determined. When the number of decomposition level is more than 9, the output CNR and the correlation coefficient are gradually stable. The bigger the levels of wavelet decomposition for the deep space weak signal are, the better the performance of wavelet extracting weak signals is. But from the simulations, it can be concluded that it is not always good when the number of decomposition layer is more than 9 since more difficulties will be taken to the deep space communication system, such as increasing the calculation.

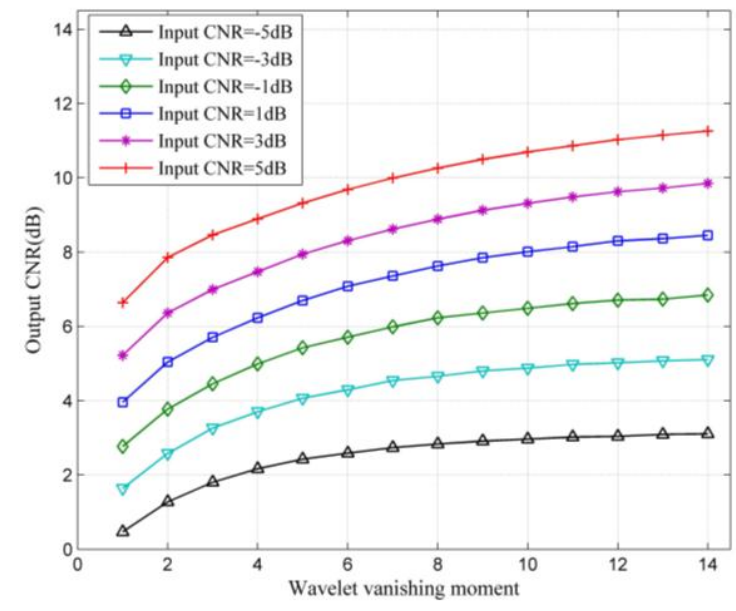

Fig. 1: The effect of wavelet function vanishing moment on the CNR of output signal.

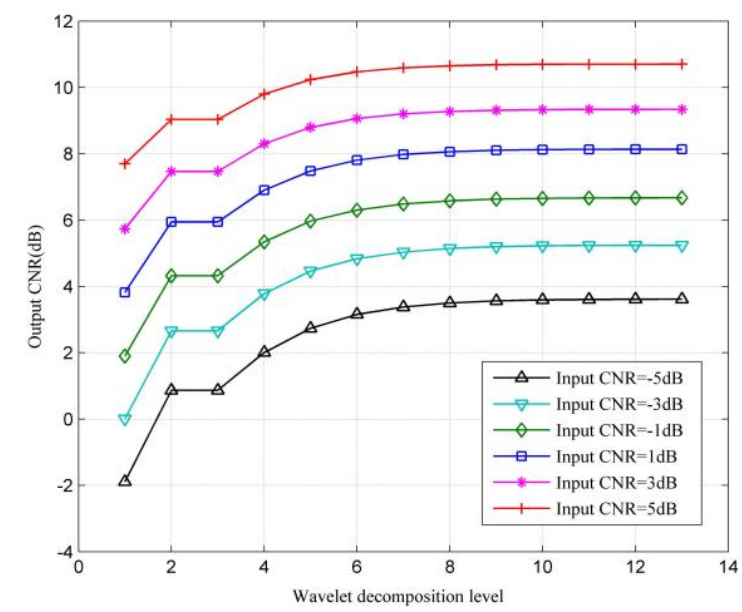

Fig. 3: The effect of the wavelet decomposition level on the output CNR.

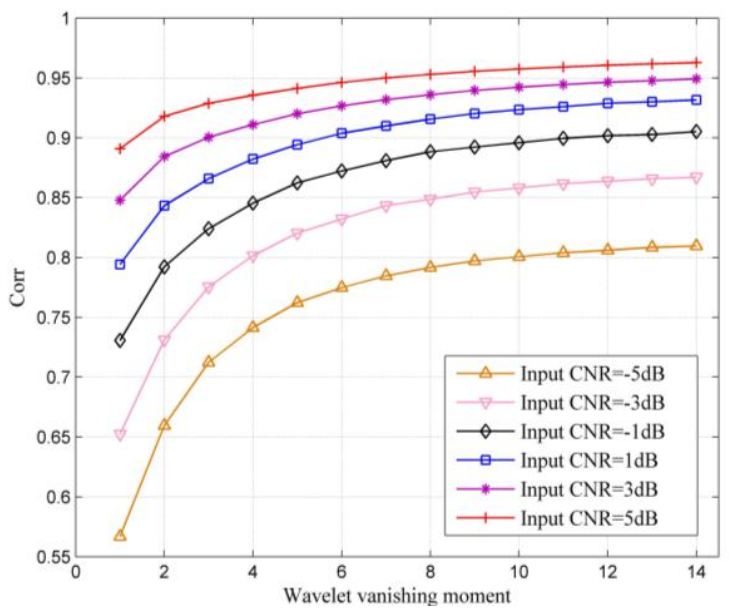

Fig. 2: The influence of vanishing moment of wavelet function on correlation coefficient.

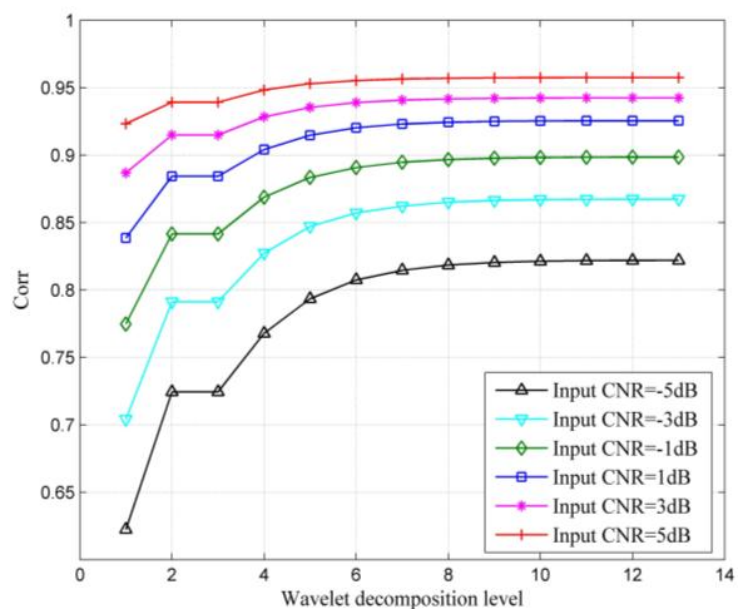

Fig. 4: The effect of the wavelet decomposition level on the correlation coefficient.

\section{Conclusions}

This paper has analyzed the wavelet threshold method for extracting weak signals in deep space communication and also gives suggestions on the selection of the optimal threshold. It has discussed the simulation results on vanishing moment of wavelet function and the wavelet decomposition level. Based on the discussions, it has concluded that the optimum vanishing moment of wavelet function is 10 and the optimum number of decomposition level is 9 in order to get the maximum gain with the least cost, which is pretty suitable for deep space communication. When deep space missions need use the wavelet to extract weak signals, we can refer to this paper to design the whole deep space communication system. Moreover, it has given some references on other super-far-distance communication when applicable for practical engineering. 


\section{Acknowledgements}

The authors would like to thank the National Natural Science Foundation of China (61571316) for the support.

\section{References}

[1] Wang C, Wang W, Sohraby K, et al. Deep space communication relay services under energy constraint with optimal power control. Global Communications Conference (GLOBECOM), 2013 IEEE., 2013, pp. 1669-1674.

[2] Wang J, Zhao P, Liang D, et al. Poster: A multi-path routing algorithm for deep space communication. 2014 9th International Conference on Communications and Networking in China (CHINACOM). IEEE Computer Society, 2014, pp. 640-641.

[3] Wu T, Yan Y, Li Y, et al. Deep Space Communication Channel Characteristics under Solar Scintillation. Instrumentation and Measurement, Computer, Communication and Control (IMCCC), 2014 Fourth International Conference on. IEEE. 2014, pp. 510-515.

[4] Shi X, Wang Y, Dang H. Study on Array Signal Processing for Deep Space Communications. Second International Symposium on Intelligent Information Technology Application. IEEE Computer Society. 2008, vol. 1, pp. 611-615.

[5] Shang Y, Feng X. MLC-SUMPLE Algorithm for Aligning Antenna Arrays in Deep Space Communication. Aerospace and Electronic Systems IEEE Transactions on. 2013, vol. 49, pp. 2828-2834.

[6] William R, Stephen D, Charles T, Senior M, Miles K. Ground Antennas in NASA's Deep Space Telecommunications. Proceedings of IEEE. 1994, vol. 82, pp. 636-645.

[7] Kay S, Nagesha V. Methods For Chaotic Signal Estimation. Signal Processing IEEE Transactions on. 1995, vol. 43, pp. 2013-2016.

[8] Bao-Qin Wang. The Mallat Algorithm for a Class of Orthogonal Wavelet on Compact Lie Groups. Wavelet Analysis and Pattern Recognition, BaoDing. 2009, pp. 413-416.

[9] Thepade S D, Bidwai P. Iris recognition using fractional coefficients of transforms, Wavelet Transforms and Hybrid Wavelet Transforms. Control Computing Communication and Materials (ICCCCM), 2013 International Conference on. IEEE. 2013, pp. 1-5.

[10] Philip B, Afroz Z. A Compact, Broadband Antenna for Planetary Surface-to-Surface Wireless Communications. Microwave and Optical Technology Letters. 2006, vol. 48, pp. 521-524.

[11] Sun T Y, Liu C C, Tsai T Y, et al. Threshold exploration via particle swarm optimizer at profitable wavelet decomposition for noise reduction. Systems, Man and Cybernetics, 2008. SMC 2008. IEEE International Conference on. IEEE. 2008, pp. 2652 - 2657.

[12] Imah E M, Al Afif F, Fanany M I, et al. A comparative study on Daubechies Wavelet Transformation, Kernel PCA and PCA as feature extractors for arrhythmia detection using SVM. TENCON 2011 - 2011 IEEE Region 10 Conference. 2011, vol. 58, pp. 5-9.

[13] Qi Haibing. Discrete Wavelet Soft Threshold Denoise Processing for ECG Signal. Intelligent Computation Technology and Automation. 2010, vol. 5, pp. 126-129.

[14] Smith C B, Agaian S. Akopian D. A Wavelet-Denoising Approach Using Polynomial Threshold Operators. IEEE Signal Processing Letters. 2008, vol. 15, pp. 906-909.

[15] Li J, Cheng C, Jiang T, et al. Wavelet de-noising of partial discharge signals based on genetic adaptive threshold estimation. IEEE Transactions on Dielectrics and Electrical Insulation. 2012, vol. 19, pp. 543-549. 